\title{
THE STRUCTURE OF COMPLETE MANIFOLDS OF NONNEGATIVE CURVATURE
}

\author{
BY JEFF CHEEGER ${ }^{1}$ AND DETLEF GROMOLL ${ }^{2}$ \\ Communicated by Stephen Smale, June 11, 1968
}

0. In this paper we describe some results on the structure of complete manifolds of nonnegative sectional curvature. (We will denote such manifolds by $M$.) Details and related results will appear elsewhere. Our work generalizes that of Gromoll, Meyer [2] as well as Toponogov [4].

Our analysis is divided into two parts. The first, which we feel is of particular interest, essentially reduces the study of the topology of complete manifolds of nonnegative curvature to that of compact manifolds of nonnegative curvature. The second step reduces the compact case to the compact simply connected case (modulo the classification of compact flat manifolds).

Our results have various applications. One of these is the classification up to isometry of complete 3-dimensional manifolds of nonnegative curvature.

1. The basic notion we use is that of a totally convex set (t.c.s.).

Definition 1.1. A subset $C$ of a riemannian manifold will be called totally convex if for any $p, q \in C$ and any geodesic $\gamma$ from $p$ to $q$, we have $\gamma \subset C$.

The significance of total convexity lies in the fact that, topologically, a t.c.s. is very similar to the manifold which contains it. Clearly, any riemannian manifold is a totally convex subset of itself. On the other hand, a point is not totally convex unless $M$ is contractible. In general, nontrivial totally convex sets do not exist. However, in [2] a "basic construction" is given which associates to each point $p$ in a complete noncompact manifold of positive curvature, a compact t.c.s. $C(p)$ containing $p$. Our fundamental observation is that by use of a different argument involving Toponogov's theorem on geodesic triangles, this construction may be carried out in the case where the curvature of $M$ is nonnegative. More generally we have:

LEMma 1.1.

(1) A closed t.c.s. is a totally geodesic submanifold (whose boundary

\footnotetext{
${ }^{1}$ N. S. F. Postdoctoral Fellow.

${ }^{2}$ Miller Fellow.
} 
is smooth almost everywhere). With $M$ as above there exists a family $C_{t}$ (for all $t \geqq 0$ ) of compact totally convex sets such that:

(2) $t_{2}>t_{1}$ implies $C_{t_{2}} \supset C_{t_{1}}$ and if $p \in \partial C_{t_{1}}$ then $\rho\left(p, \partial C_{t_{2}}\right)=t_{2}-t_{1}$,

(3) $U_{t} C_{t}=M$,

(4) $\operatorname{dim} C_{0}<\operatorname{dim} M$.

Let $C^{a}=\{p \in C \mid \rho(p, \partial C)-\geqq a\}$.

Lemma 1.2. If $C \subset M$ is a t.c.s. then for any $a$, so is $C^{a}$. Letting $C^{\max }$ denote the subset of points at maximum distance from $\partial C$ (in the sense of (1) in Lemma 1.1) one has $\operatorname{dim} C^{\max }<\operatorname{dim} C$.

Lemma 1.1 together with the iteration of Lemma 1.2 and some Morse Theory yield:

THEOREM 1.3. There exists a t.c.s. $S(M) \subset M$ which is a compact totally geodesic submanifold without boundary (and has nonnegative curvature). The inclusion $S(M) \hookrightarrow M$ induces isomorphisms $\pi_{i}(S(M)$ ) $\rightarrow \pi_{i}(M)$ for all $i$, and hence is a homotopy equivalence.

We call $S(M)$ the soul of $M$. Any soul (in general the constructions of Lemma 1.1 depend on the choice of some arbitrary $p \in M$ ) is clearly a minimal t.c.s.

THEOREM 1.4. $M$ is diffeomorphic to the normal bundle $\nu(S(M))$ of $S(M)$ in $M$. All curvatures with respect to planes spanned by a tangent and a normal vector vanish along $S(M)$.

TheOREM 1.5. If $\nu(S(M))$ is orientable and $\operatorname{dim} S(M)=\operatorname{dim} M-1$ then $M$ is isometric to $S(M) \times R$ (with the product metric).

2. The following theorem is the basic result in the compact case.

THEOREM 2.1. Let $M$ be a compact manifold of nonnegative curvature, then there is a diagram of covering maps,

$$
\begin{aligned}
\tilde{M} & \stackrel{i_{2}}{\leftarrow} M_{0} \times R^{k} \\
\pi_{2} & \downarrow \\
\tilde{M} & \stackrel{i_{1}}{\leftarrow} M_{0} \times F \\
\pi_{1} & \downarrow \\
M &
\end{aligned}
$$

such that

(1) $\pi_{i}$ are local isometries,

(2) $M_{0}$ is a compact simply connected manifold of nonnegative curvature and $i_{2}$ is an isometry, 
(3) $F$ is a compact flat manifold and $i_{1}$ is a diffeomorphism,

(4) $\pi_{1}$ is a finite covering.

Theorem 2.1 depends on the result of Toponogov given in [4]. Toponogov's result on the other hand, is an easy consequence of our "basic construction."

3. We sketch some applications of the above results.

(A) The following provides at least a partial answer to a generalization of a problem of Chern [1]. The original problem was solved in $[2]$.

THEOREM 3.1. With the notation of Lemma 1.1:

(1) If $\gamma:(-\infty, \infty) \rightarrow M$ is a geodesic then at least one end of $\gamma$ goes to infinity or $\gamma \subset \partial C_{t}$ for some $t$.

(2) Any geodesic intersecting $S(M)$ transversally goes to infinity in both directions.

(3) If $\sigma, \tau:[0, \infty) \rightarrow M$ are geodesics, $\sigma(0)=\tau(0),\left\langle\sigma^{\prime}(0), \tau^{\prime}(0)\right\rangle>0$ and $\sigma$ is a ray then $\tau$ goes to infinity.

(B) If $\operatorname{dim} S(M)=1$ then the universal covering space $\tilde{M}$ is isometrically $\bar{M} \times R$ where $S(\bar{M})$ is a point. This follows from the result of Toponogov [4].

This remark together with Theorem 1.5 forms the basis for the classification up to isometry of complete noncompact 3-manifolds of nonnegative curvature. The solution of the classification problem in two dimensions is classical and due to Cohn-Vossen.

(C) Theorem 1.3 and Theorem 2.1 yield the following result, the first part of which is a generalization of a result of Milnor [3] in our situation:

THEOREM 3.2. Modulo some finite invariant subgroup, the fundamental group of a complete (compact or not) manifold $M$ of nonnegative curvature is a finite extension of the free abelian group $\boldsymbol{Z} \oplus \cdots \oplus \boldsymbol{Z}$ with $k$ generators, $0 \leqq k \leqq \operatorname{dim} M$. The higher homotopy groups are finitely generated. $\pi_{1}(M)$ infinite implies that the Euler characteristic $\chi(M)$ vanishes.

(D) Theorem 1.3 and Theorem 1.4 can be used to show that most spaces do not admit any complete metric of nonnegative curvature.

(E) Theorem 1.3 and Theorem 1.4 may be applied to the case of homogeneous spaces and flat manifolds to yield isometric decompositions which in some cases do not seem to appear in the literature. Theorem 2.1. on the other hand may be used to show that a metric of nonnegative curvature on a manifold diffeomorphic to a compact 
flat manifold is necessarily flat. This answers a conjecture of Auslander and Wolf posed in [5].

(F) The notion of totally convex sets can be used to study isometries of complete manifolds of nonnegative curvature.

\title{
BIBLIOGRAPHY
}

1. J. Eells and S. Kobayashi, Problems in differential geometry, Proceedings U. S.Japan Seminar on Differential Geometry, Kyoto (1965), pp. 167-177.

2. D. Gromoll and W. Meyer, On complete open manifolds of positive curvature, University of California, Berkeley, Preprint; Ann. of Math. (to appear).

3. J. Milnor, A note on curvature and fundamental group, J. Differential Geometry (to appear).

4. V. Toponogov, Spaces with straight lines, Amer. Math. Soc. Transls 37 (1964), 287-290.

5. J. Wolf, The rate of growth of discrete solvable groups and its influence on the curvature of riemannian manifolds with solvable fundamental group, University of California, Berkeley; J. Differential Geometry (to appear).

University of California, Berkeley

\section{ON MIXING IN INFINITE MEASURE SPACES}

\author{
BY U. KRENGEL AND L. SUCHESTON ${ }^{1}$
}

Communicated by David Blackwell June 17, 1968

1. E. Hopf $[6$, p. 66] suggested that strong mixing in infinite measure spaces should be defined by a limit statement on certain ratios; Krickeberg [9] made this precise in the context of topological measure spaces. In this paper we shall consider a different concept of strong mixing, meaningful also without existence of a topological structure. Our notion coincides with the usual concept of strong mixing in the case of finite measure spaces and seems to be the proper generalization to the infinite measure case in that it is exactly the concept needed to carry over certain theorems on mixing which hold in finite measure spaces.

Given a sequence $\left(A_{n}\right)$ of measurable sets on a measure space $(\Omega, Q, \mu)$, the intersection $R\left(A_{n}\right)$ of the $\sigma$-algebras $B_{k}\left(A_{n}\right)$ generated by $A_{k}, A_{k+1}, \cdots$ will be called the remote $\sigma$-algebra of $\left(A_{n}\right)$. A sequence $\left(A_{n}\right)$ is called remotely trivial, iff $\mathcal{R}\left(A_{n}\right)$ is trivial, i.e., contains only

\footnotetext{
${ }^{1}$ Research of this author is supported by the National Science Foundation under Grant GP7693.
} 